\title{
Distance perception under binocular and monocular viewing conditions
}

\author{
NANCY PARKER LEVINE and RICHARD R. ROSINSKI \\ University of Pittsburgh, Pittsburgh, Pennsylvania 15260
}

\begin{abstract}
First, third, and fifth graders and college adults made judgments of absolute distance in a binocular (full-information) condition and in one of three monocular conditions: redundant texture gradient, compression gradient, and control (no texture). No age-related differences in accuracy of judgment were observed in any of the conditions. Substantial differences in the effectiveness of different kinds of information were found, however. The results indicate that the ability to register information for distance is well developed by first grade, but that substantial limitations exist on the visual system's ability to process various forms of redundant information.
\end{abstract}

Previous research into the development of space perception has suggested that some components of the ability to perceive spatial layout are present in early childhood. In particular, some studies have also demonstrated that infants are able to perceive depth and distance on the basis of binocular stereopsis or motion perspective. For example, Appel (1971) has suggested that infants can register binocular disparity information which may allow the localization of an object in space. even in the absence of any other visual information. Campos. Langer, and Krowitz (1970) measured heart rate while the infant was placed over the deep or shallow side of a visual cliff. Their results showed that children under the age of 2 months are able to discriminate between the two depths of the cliff. In addition. the original work of $W$ alk and Dodge (1962) and Walk and Gibson (1961) demonstrates that older infants can use motion perspective information for the accurate perception of depth.

Other aspects of space perception, specifically those involving the use of static monocular information, appear to have a much longer developmental course. Bower $(1964,1965)$ found that although infants were able to respond consistently to the distance and size of an object under conditions which allowed binocular parallax or motion perspective, they were unable to do so when distance and size were specified only by static monocular information. In addition. Bower (1966) also found that 2 -month-old infants were unable to

Based on a dissertation by the first author, submitted in partial fultillment of the requirements for a PhD degree at the University of Pittsburgh. This research was supported, in part, by a grant from the National Institute of Child Health and Human Development (1-R01-HD07307) to Richard R. Rosinski. The authors wish to thank Maryann Bishop, Bruce Goldstein, Lynn Katz, and Alex Siegel for their comments on the manuscript, and Karen Kukish and Carol Wildemann for assistance in conducting the experiment and analyzing the data. Requests for reprints should be sent to Richard R. Rosinski. Department of Psychology, University of Pittsburgh, Pittsburgh. Pennsylvania 15260. respond to surface slant. which is exactly specified by texture gradient information. Thus Bower's results suggest that infants may be deficient in the ability to use static monocular information.

A number of other studies have attempted to assess children's ability to perceive spatial layout on the basis of monocular information. Yonas and Hagen (1973) used children's judgments of size to evaluate the effectiveness of texture information and height in the lield. and found that both of these variables signiticantly influenced 3-year-olds' size judgments. However. since an object must occlude a constant number of texture elements independent of its distance from the observer, accurate size judgments could be based on the number of texture elements occluded rather than on the perception of depth.

Wohlwill (1965), using a bisection procedure. found that texture gradient information influenced children's judgments of relative distance. Similarly, McGurk and Jahoda (1974) and Olson (1975) evaluated children's sensitivity to pictorial cues for distance. Both studies demonstrated that children 3 and 4 years old were able to make relative judgments of distance by indicating which of two objects was farther away and both found angular height in the field to be an important cue for judgments.

Although these studies show that children are able to register (or interpret) some pictorial cue for relative distance, they do not directly bear on child ren's ability to perceive absolute distance based on monocular information.

The present study measured children's ability to perceive absolute distance under conditions in which specitiable manipulations of texture gradient information were introduced. Purdy (Note 1) has shown that the texture gradients of perspective, compression, size, and density, projected to any station point, provide information for the perception of actual distance and slant, since gradient information is in one-to-one correspondence with distance and surface slant. Research on the 
monocular perception of slant (Rosinski \& Levine, 1975; Degelman \& Rosinski. Note 2) has shown that first grade children are sensitive to texture gradient information and can make judgments of surface slant based solely on such information. Both studies found improvements in performance with age which did not depend on an improvement in the ability to differentiate texture gradient information. but which suggested an improvement in the ability to relate texture gradient information to a geographical coordinate system. Judgments of distance based on texture gradient information do not require that gradients be related to geographical coordinates in the way that slant perception does. Consequently, if children are able to accurately extract texture gradient information as the previous slant studies suggest. they should be able to accurately judge distance.

Rosinski and Levine (1976) also found that theoretically equivalent sources of texture gradient information were differentially effective in perception. For example, texture gradient information provided by element compression (foreshortening) was virtually ineffective compared to information provided by size and perspective. If such differences in information effectiveness reflect differences in basic processing abilities, as suggested by Rosinski and Levine, similar differences in the effectiveness of gradient information may be expected in the judgment of distance.

The present study manipulated texture gradients which provide theoretically sufficient information for distance. The purposes of the study were fourfold: tirst. to determine whether children can accurately perceive distance using texture gradient information; second, to determine whether certain sources of texture gradient information are more effective in perception than others; third, to determine the effectiveness of angular size and elevation cues relative to the effectiveness of texture gradient intormation; and fourth, to compare accuracy when distance is specified by binocular stereopsis. motion parallax, and texture gradient information vs. when distance is specitied by angular elevation and size alone.

\section{METHOD}

\section{Subjects}

A total of 96 subjects were tested: 24 from each of first (mean age 6.9 years). third (mean age 8.5 years), and fifth (mean age 11.1 years) grades. and 24 college student participants from the University of Pittsburgh. An equal number of male and female subjects were assigned to each condition at the fitth grade and adult levels. The sex ratio in the particular school did not allow such equal assignment in the first and third grades. Consequently, an equal number of female subjects were assigned to each of the experimental conditions in these two grades.

\section{Design}

Each subject made judgments of absolute distance under conditions of full binocular viewing, and in one of three monocular viewing conditions (no texture information; compression gradient information; or redundant gradient information provided by size. density, perspective, and compression). These monocular conditions were used to allow comparisons to be drawn between the present study and the previous slant studies, as well as to allow the evaluation of the effectiveness of the no-gradient condition. The presentation order of the monocular and binocular conditions was counterbalanced so that each subject served as his own control.

In each of the two viewing conditions, each subject made 18 judgments in three blocks of six distances for a total of 36 judgments per subject.

\section{Apparatus}

Target. In each condition, subjects judged the distance of a small irregular white target outlined in black. The target was constructed using modified Attneave and Arnoult (1956) techniques; seven points were randomly generated on a $10 \times 10 \mathrm{~cm}$ matrix, and the points were connected to form a closed area. Each of the angles thus formed was then rounded off to a randomly determined radius.

Monocular background surfaces. The three monocular stimulus displays were created by photographing the target on one of three background surfaces. A white Foamcore surface, $76 \times 305 \mathrm{~cm}$ in size, was oriented horizontally. On this surface, two black fixed reference stripes, $10 \mathrm{~cm}$ thick, were positioned. These stripes extended the entire width of the surface and were located $140 \mathrm{~cm}$ apart. A sheet of white, seamless background paper was positioned perpendicularly behind the surface to eliminate any other information and to provide an artiticial horizon.

Examples of the three monocular stimulus conditions are presented in Figure 1. For the control (no-texture) condition, the surface was used as described above. For the compression gradient condition. $1.9-\mathrm{cm}$ alternating black and white stripes were spaced across the width of the entire surface. For the multiple gradient condition. $1.9-\mathrm{cm}$ black and white stripes were positioned across the surface's width and length providing the grid of $1.9-\mathrm{cm}$ squares.

In preparing the monocular displays. the target was placed at one of six distances $(20,40,60,80.100$, or $120 \mathrm{~cm})$ from the reference stripe nearest the camera.

Photographic conditions. The stimulus surfaces were photographed with a $4 \times 5$ view camera with a 215 -mm lens. When all camiera adjustments were zero, the surface was horizontal. the film plane of the camera was vertical, and the axis of the lens was $39 \mathrm{~cm}$ above the Foamcore surface. In order to take the photographs, the back standard was raised $4 \mathrm{~cm}$. the front standard was lowered $4 \mathrm{~cm}$, and the film plane was kept vertical. The front standard was then tilted $18 \mathrm{deg}$ in order to maximize depth of field by the Scheimptlug effect. After focusing, the nodal front of the lens was $23 \mathrm{~cm}$ away from the film plane measured along the perpendicular to that plane. Photographs were taken on Polaroid 55P $\mathrm{N}$ film, and the negatives were entarged exactly two times and printed on Agfa Fo paper, which was normally processed and dried to a matte tinish. The photographs were then mounted on heavy matte board.

Monocular viewing apparatus. For the monocular viewing conditions, each matted photograph was placed in a viewbox. $27.5 \mathrm{~cm}$ wide $\times 43 \mathrm{~cm}$ long $\times 37.5 \mathrm{~cm}$ high. A viewing aperture. $6.4 \mathrm{~mm}$ in diam. fixed the subject's eye at the correct station point. The viewing point was $16 \mathrm{~cm}$ above the center of the photograph measured parallel to the plane of the photograph and $46 \mathrm{~cm}$ away from the photograph measured perpendicular to the plane. This placement of the viewing point resulted in an optic array to the eye which was geometrically identical to the array available to a subject actually viewing the stimulus surfaces. The interior of the box was painted a tlat black and a $40-\mathrm{W}$ incandescent lamp was placed inside the box to illuminate the photograph. The lamp was placed in a cylindrical Polaroid tilter. and a second. cross-polarized filter was placed over the viewing aperture to eliminate any glare from the surface of the photograph.

Binocular stimulus display. The binocular display consisted of a gray Masonite surface. $38 \mathrm{~cm} \times 155 \mathrm{~cm}$. with two $10-\mathrm{cm}$ black 
reference stripes $140 \mathrm{~cm}$ apart on the surface placed before the subject. Lnder the binocular testing conditions. the target was placed at one of the six distances on th is binocular display surface. Each subject looked directly at the display with binocular vision and tree head movement.

Response apparatus. Judgments of distance were made using a reproduction procedure. Each subject made his distance judgments on an apparatus similar to the one used as a binocular display. A white target. identical to the one used in the monocular and binocular display conditions. was moved between the two stripes by means of a pulley system attached to a crank near the subject's hand. The response apparatus was positioned so that the subject's view of it was similar to his view of the monocular and binocular stimulus displays and at a right angle to them. The relative position of the station point (in terms of height and angular sizes) was similar for the monocular and binocular displays and for the response apparatus. While responding, subjects were allowed binocular vision and unrestrained head motion.

\section{Procedure}

Each subject was tested individually while in a chair which was adjusted so that he could view the response and stimulus displays from the appropriate height. Each subject was first instructed in the use of the response device. and was given two practice trials in which he was asked to set the target so that it was the same distance away as the experimenter's finger placed on the response device.

In the instructions for both the binocular and monocular viewing conditions, the subject's attention was drawn to the black stripes on the stimulus displays and the response surface. He was told that the reference stripes were the same in both cases and instructed to position the white target on the response apparatus in the same place as the white target in the stimulus display.

Subjects were allowed to view the stimulus displays as long or as often as they wished and to alternate between the stimulus displays and the response surface as often as wished. Once the subject completed his response. the experimenter recorded the distance to the nearest centimeter. the target was placed at one end (randomly determined) of the response apparatus. and the next trial began.

\section{RESULTS}

Analyses were conducted on subjects judgments of distance recorded in centimeters. Since no sex differences were found. $t(190)=-.183, \mathrm{p}>.05$, the data were pooled across the variable for subsequent analyses. A number of analyses were conducted to evaluate subjects performance in the monocular condition. in the binocular condition. and the two conditions compared.

\section{Monocular Viewing Conditions}

Distance judgments in the monocular viewing conditions were analyzed using a 4 (grade) by 3 (gradient condition) by 3 (block) by 6 (distance) analysis of variance with repeated measures on the last two factors. Figure 2 depicts the subject's judgment of distance as a function of monocular condition. There was no significant difference among grades in the monocular viewing task, $F(3,84)=.82$, $\mathrm{p}>.10$, indicating that over the age range studied there was no improvement in the ability to differentiate texture gradient information for distance. As predicted from the results of the slant studies. the different information in the monocular
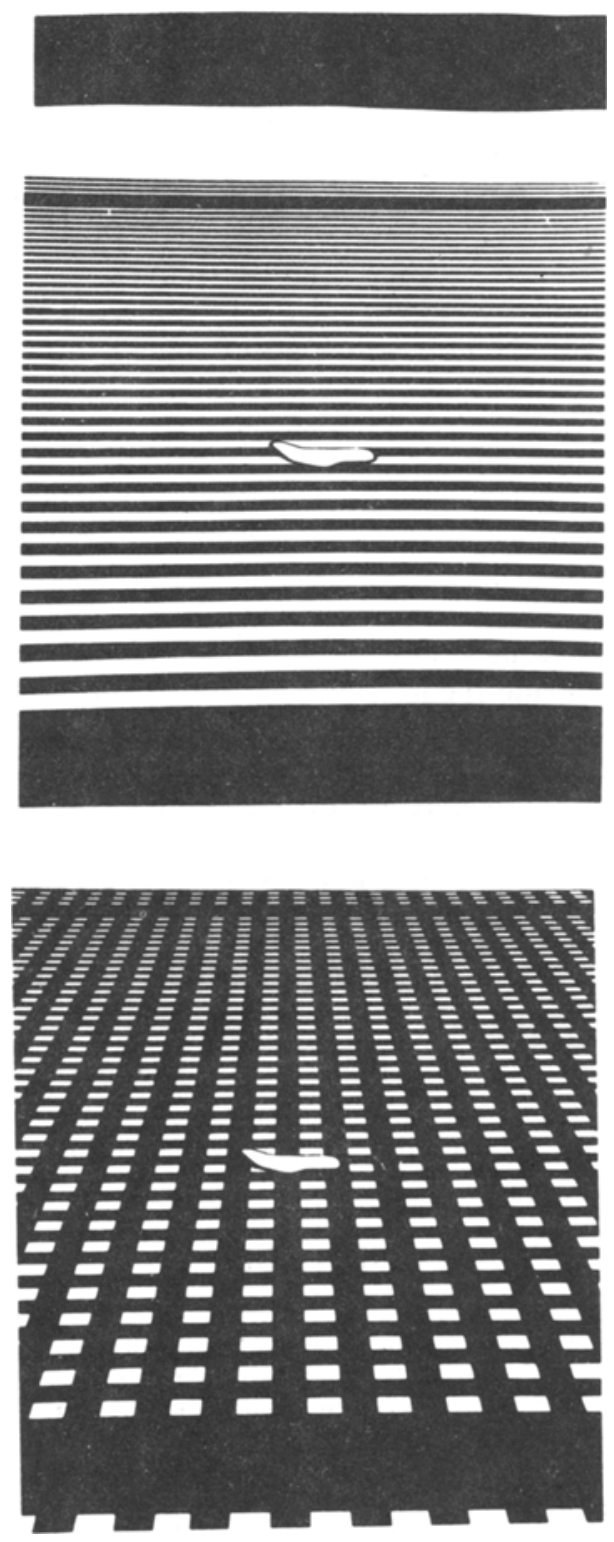

Figure 1. Sample monocular stimulus displays in the combination gradient, compression gradient, and control conditions. 


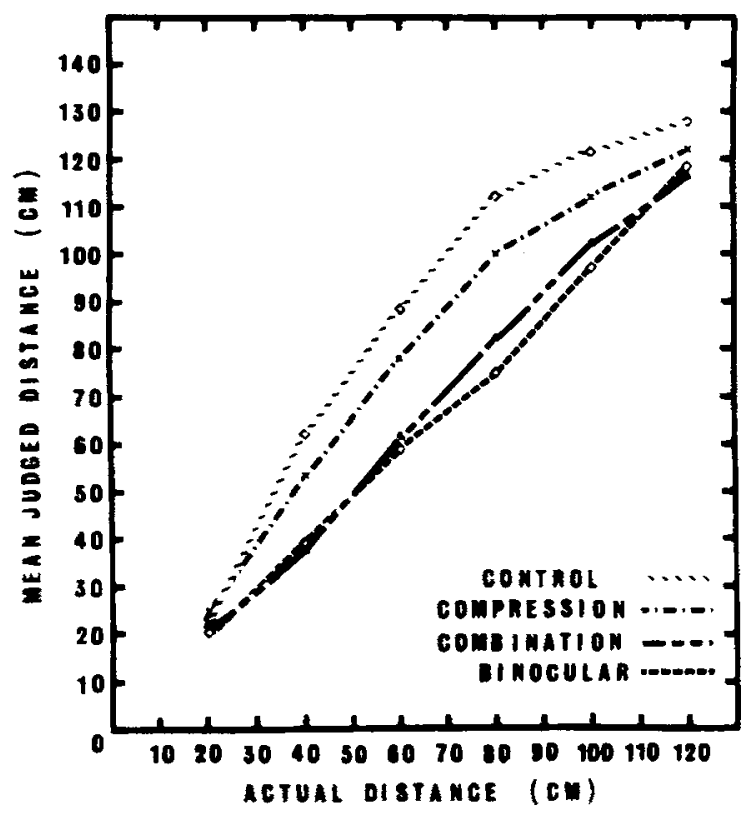

Figure 2. Mean Judged distance as a function of actual distance for the binocular viewing condition and each of the monocular conditions.

viewing conditions provided differentially effective bases for distance judgment. $F(2,84)=94.71$, $\mathrm{p}<.001$. Individual comparisons showed that judgments in the combination gradient condition (the grid) were significantly different from those in the compression gradient (horizontal lines) condition, which were significantly different from those in the control condition (all ps $<.01$ ). An indication of the accuracy of judgment under these three monocular viewing conditions is given by comparing subjects mean judgments across all physical distances with the mean distance represented in the study. The mean of the six distances used in the study was $70 \mathrm{~cm}$, and the mean judgment for the combination gradient condition was also $70 \mathrm{~cm}$. The mean judgment of distance in the compression gradient condition. however. was $82 \mathrm{~cm}$. and the mean in the no-texture condition was $89 \mathrm{~cm}$. These data show considerable overestimation of the distance by subjects in the latter two conditions, and show that such overestimation is greater in the control condition in which angular size and angular elevation provided the only source of information for distance.

The effect of block was not significant, $F(2,168)=$ .78. $p>.10$. indicating that judgments of distance did not improve with short-term practice during the experiment. As is to be expected, there were signiticant differences among distance, $F(5.420)=$ $1.859 .66 . p<.001$. showing that subjects' judgments of distance were related to physical distance. That is. $20 \mathrm{~cm}$ was judged to be significantly different from 40 cm. etc.
As can be seen in Figure 2. there was a signiticant interaction between monocular condition and distance of the target, $F(10,420)=13.32, p<.001$. This effect suggests that reproduction judgments of distance are easiest near the black reference stripes in all conditions. As can be seen from the figure. subjects' judgments of distance near these anchor points (e.g., 20 and $120 \mathrm{~cm}$ ) were quite accurate under all three monocular conditions. However, as target distance departed from the reference stripes. inaccuracies in judgment resulted whicb were differentially reflected in the three monocular conditions. No increase in error was observed in the multiple gradient condition, but considerable overestimation took place in the other conditions. As Table 1 shows, the subjects judgments in the control (no-gradient) condition closely followed a line predicted on the basis of visual angle estimations. The visual angle predictions at each distance lie within the .05 confidence intervals for judgments in the control condition. Consequently. although subjects' judgments are in some correspondence with actual distance. their judgments suggest that they are directly mapping angular separation between target and reference stripes into distance. While such use of elevation is sufficient for relative judgments and allow's the subject to make some estimation of distance, it results in sizable errors of distance estimation. especially in the middle range of distances where visual angles and physical distances are not isomorphically related. No other main effects or interactions were significant, all ps $>.10$.

To further evaluate the relationship between judgment and actual distance, individual Pearson product-moment correlations were computed and averaged by means of Fisher $\mathrm{Z}$ transformations. Across all grades, the correlations for the three monocular viewing conditions were very high and quite similar: .93 for the combination gradient condition, 91 for the compression gradient condition, and .95 for the no-gradient condition. These correlation coefficients show a strong linear relationship between judged and physical distance

Table 1

Performance in Control Conditions Compared to Visual Angle Predictions

\begin{tabular}{cccc}
\hline Actual & Mean Judged & $\begin{array}{c}\text { Visual Angle } \\
\text { Predictions } \\
\text { Distances } \\
(\mathrm{cm})\end{array}$ & $\begin{array}{c}\text { Predicted on } \\
\text { Basis of Visual } \\
\text { Dinus Mean }\end{array}$ \\
$\begin{array}{c}\text { Angle }(\mathrm{cm}) \\
\text { Judgments } \\
(\mathrm{cm})\end{array}$ \\
\hline 40 & 23 & 32 & +9 \\
60 & 62 & 70 & +8 \\
80 & 89 & 94 & +5 \\
100 & 112 & 109 & -3 \\
120 & 121 & 122 & +1 \\
\end{tabular}


under all monocular viewing conditions. It is especially important to note that the control condition, which provided only visual angle cues for distance, resulted in a high correspondence between judged and physical distance. This strong relationship again shows that visual angle cues provide information for judgments of distance, even though these judgments were less accurate than in the texture gradient conditions.

\section{Binocular Viewing Condition}

The data for the subjects judgments in the binocular viewing task across the six distances are also presented in Figure 2. Subjects' judgments were analyzed in a 4 (grade) by 3 (block) by 6 (distance) analysis of variance with repeated measures on the last two factors. As in the monocular viewing task. there were no significant effects of grade, $F(3,28)=$ $.33, \mathrm{p}>.10$. The mean judgments for the four grades were within approximately $1 \mathrm{~cm}$ of one another and only slightly below the actual mean.

Block was also not a significant factor, $F(2.56)=$ 2.23, $\mathrm{p}>.05$, again showing that practice did not affect judgments in the course of this experiment. A significant difference among distances, $F(5.140)=$ $1,083.33, \mathrm{p}<.001$, shows that subjects' responses were closely related to the actual distance presented. Only one interaction was found to be significant. Grade by Block, $F(6,56)=3.41, p<.01$. The data indicate that this interaction is due to the effect of block (i.e., practice) at only the fifth grade level. This result is not readily interpretable, and may be due to particular characteristics of the sample chosen. No other interactions were significant (all ps $>.10$ ).

Mean Pearson product-moment correlation coefficients ranged from .95 to .98 for the four grade levels. showing a close correspondence between judged and physical distance at all grade levels in the binocular condition.

\section{Monocular vs. Binocular Conditions}

Since each subject served as his own control in that each participated in the binocular viewing condition and in one of the monocular viewing conditions, monocular-binocular comparisons were made in terms of three within-subject analyses of variance comparing subject's performance in each of the monocular viewing conditions with their performance in the binocular condition. In the comparison between the binocular viewing condition and the monocular viewing condition involving the combination (grid) gradient, neither the effect of grade, $F(3,28)=.10$, $\mathrm{p}>.10$. nor the effect of viewing task, $F(1,28)=$ $4.10 . \mathrm{p}>.05$, was significant. These results indicate no difference in accuracy of absolute judgment when distance is specified by multiple redundant information, including binocular disparity and motion parallax information. or when distance is specitied only by the four monocular texture gradients.

In the comparison between the binocular viewing condition and monocular compression gradient condition, there was also no effect of grade, $F(3,28)=$ .52 . $\mathrm{p}>.10$. However, there were substantial differences between the viewing tasks, $F(1,28)=$ 91.23, $\mathrm{p}<.001$. Subjects were considerably less accurate when distance was specified by angular cues and compression gradient information than when distance was specified by all potentially available information. This same pattern of results was found in the comparison between binocular and control conditions. Again, grade was not a significant factor, $F(3.28)=1.18, p>.10$, but substantial differences in accuracy as a result of viewing task were found, $F(1.28)=1.057 .88, p<.001$. Specification of absolute distance only in terms of angular size and angular elevation cues resulted in substantially poorer performance than in the binocular situation.

\section{DISCUSSION}

Our results indicate that the ability to make judgments of absolute distance is highly developed by the first grade. Performance at even the youngest age level demonstrated a close correspondence between physical and judged distance. In addition, no improvement in accuracy of judgment is seen over the age span covering first graders and college students. If the ability to register and use various kinds of distance information does, in fact, change with development. it must do so early in childhood, and for a wide range of optical stimulation specifying distance. Regardless of the kind of information (binocular disparity, motion perspective, linear perspective, multiple texture gradients. compression gradients, or pictorial cues), no developmental changes in judgmental accuracy were observed. Although Wohlwill's (1965) results suggested some developmental improvement in distance perception, our data support Smith and Smith's (1966) argument that this improvement is an artifact of the bisection procedure.

Performance in the multiple texture gradient condition was statistically indistinguishable from performance based on the combination of binocular disparity, motion perspective, and linear perspective. This is, in itself. a most surprising finding, for it suggests that the various subsystems involved in space perception are equivalent in accuracy. The addition of potential binocular and motion-carried information does not improve performance.

In addition, these results bear on registration and use of texture gradient information in the perception of spatial layout: specifically, the relationship between the role of texture gradients in the perception of distance and their role in the perception of slant. Previous slant studies (Rosinski \& Levine, 1976; 
Degelman \& Rosinski, Note 2) suggest that developmental changes in slant perception do not involve changes in the ability to register information. but rather the ability to relate texture gradient information to a geographical coordinate system.

The results of the present study support this suggestion, since. in the judgment of distance from a single station point, determining the relationship between optical information and the geographical coordinate system is not necessary. For judgments from a single station point, texture gradients projected to that station point are in unique correspondence with distance. Consequently, if a subject is able to differentiate these gradients, he possesses a sufficient basis for the judgment of distance. The lack of any developmental differences in the present study suggests that the ability to differentiate or register texture gradient information is equally well developed in subjects across our entire age range.

A second aspect of the monocular distance judgment data confirms the existence of a substantial limitation on information processing in space perception. The gradient of compression, while sufficient for accurate ordinal distance judgments, did not permit observers to make accurate absolute distance judgments. The addition of perspective, size. and density gradients in the combination condition resulted in accurate absolute distance judgments. Thus, the visual system does not make equal use of different forms of gradient information. even though they are geometrically equivalent. These results extend to judgments of distance, the results obtained by Rosinski and Levine: although perspective and multiple gradients provide highly effective information for slant judgments, compression gradients are virtually ineffective. These differences in the sufficiency of gradient information cannot be ascribed to particular tasks, displays, apparatus, or subject samples; rather, they point to a general characteristic of the visual system.

Theoretically, angular elevation in the visual field could provide information for absolute distance if angular relations were appropriately mapped into linear distance and if the perceptual system could compensate for eye position (since elevation is a joint function of distance and fixation point). Our data provide no evidence that such abilities exist either in children or adults. Rather, in the absence of other information. subjects directly map angular relation onto linear distance, such that a target which is half the angular distance between two reference points is judged as being linearly equidistant from the two points.

In summary, our results reveal a number of characteristics of the perceptual system involved in distance perception. First. the ability to perceive distance under binocular or monocular viewing conditions is apparently well developed by first grade and does not improve with subsequent experience or development. Secondly, both children and adults are equally capable of making accurate judgments based solely on static monocular information. Thirdly, the differential effectiveness of various sources of information is not restricted to particular situations or perceptual tasks; subjects are relatively poor in registering and using compression gradient information both in the perception of slant and in the perception of distance. Fourth, although others have shown that angular elevation provides a sufficient basis for judgments of relative distance, it does not result in accurate perception of absolute distance.

\section{REFERENCE NOTES}

1. Purdy, W. C. The hypothesis of psychophysical correspondence in space perception. General Electric Technical Information Series, No. R60ELC56, 1960.

2. Degelman. D., \& Rosinski, R. R. Developmental processes in the perception of slant. Paper presented at the meeting of the Midwestern Psychological Association, Chicago, May 1974.

\section{REFERENCES}

Appet. M. A. Binocular parallax in the eight-week-old infant (Doctoral dissertation. University of Denver, 1971). Dissertation Abstracts International, 7329-8. (University Microfilms No. $72-16.936$ )

Attreave, F.. \& Arnoult, M. D. The quantitative study of shape and pattern perception. Psychological Bulletin, 1956, 53. 452.471.

BowER. T. G. R. Discrimination of depth in premotor infants. Psychonomic Science, 1\%4, 1, 368.

BOwER, T. G. R. Stimulus variables determining space perception in infants. Science, 1965, 149, 88-89.

BOWER. T. G. R. The visual world of infants. Scientific American. 1\%66, 215, 80-92.

Campos, J. J., Langer, A., \& Krowitz, A. Cardiac responses on the visual cliff in prelocomotor human infants. Science. 1970, 170. 196-197.

McGurk. H., \& JaHODA, G. The development of pictorial depth perception: The role of figural elevation. British Joumal of Psychology, 1974, 65, 367-376.

Orson. R. K. Children's sensitivity to pictorial depth information. Perception \& Psychophysics, 1975. 17, 59-64.

Rosinski, R. R., \& LEVINE, N. P. Texture gradient effectiveness in the perception of surface slant. Joumal of Experimental Child Psychology, 1976, in press.

Smrrt, O. W.. \& Smrri, P. C. Developmental studies of spatial judgments by children and adults. Perceptual and Motor Skills, 1966, 22, 3-73.

WAIK, R. D., \& DodGe, S. H. Visual depth perception of a 10-month-old monocular human infant. Science, 1962, 137, 529-530.

WALr, R. D., \& Gasos, E. J. A comparative and analytical study of visual depth perception. Psychological Monographs, 1961, 75(15, Whole No. 519).

WoHLwIL, J. F. Texture of the stimulus field and age as variables in the perception of relative distance in photographic slides. Journal of Experimental Child Psychology, 1965, 2, 163-177.

Yonas. A. \& HAGEN, M. Effects of static and motion parallax depth information on perception of size in children and adults. Journal of Experimental Child Psychology, 1973, 15. 254-265.

(Received for publication May 27. 1975; revision received November 24,1975 .) 